\title{
Google News y el impacto de la Ley de Propiedad Intelectual en la prensa: un nuevo amanecer para la información
}

\author{
Juan Carlos Marcos ReCIO \\ jmarcos@ucm.es \\ Universidad Complutense de Madrid \\ Juan Miguel SÁNCHEZ VIGIL \\ jmvigil@ucm.es \\ Universidad Complutense de Madrid \\ María OLIVERA ZALDÚA \\ molivera@ucm.es \\ Universidad Complutense de Madrid
}

Recibido: $11 / 05 / 2015$

Aceptado: 12/06/2015

\section{RESUMEN}

2015 se recordará como el gran año del cambio en los medios de comunicación, especialmente para la prensa. A lo largo del mismo se ha llevado a cabo una enconada lucha entre los editores y Google News, a la que se han sumado Yahoo News, Facebook, LinkedIn, Twitter, etc., para determinar quién debe pagar por los contenidos, quién los creará, quién los distribuirá y quién y cómo los leerá. Nuevos pactos para nuevos lectores. Nuevos acuerdos para reflotar económicamente la prensa. Y todo ello bajo el paraguas de la Ley de Propiedad Intelectual en España y otras similares que se irán publicando o adaptando en Europa. Se ofrece un estudio y análisis de la situación previa al establecimiento de dicha ley y las consecuencias de su aplicación.

Palabras clave: Prensa, Medios de comunicación, Google News, Facebook, Twitter, LinkedIn, Ley de Propiedad Intelectual

\section{Google News and the Law on Intelectual Property: a new sunrise for the information}

\begin{abstract}
2015 will be remembered as the great year of change in the media, especially the press. Along the same, a close fight between newspaper publishers and Google News, which have joined other Yahoo News, Facebook, LinkedIn, Twitter, etc. To determine who should pay for content, who will create them, who will distribute them and who and how read. New agreements for new readers. New agreements to economically revive the press. And all under the umbrella of Intellectual Property Law in Spain and similar ones will be published or adapted in Europe. A study and analysis of the situation before the establishment of the law and the consequences of its application is available.

Keywords: Newspapers, Media, Google News, Facebook, Twitter, LinkedIn, Intellectual Property Law

\section{Referencia normalizada}

Marcos Recio, J.C.; Sánchez Vigil, J.M. y Olivera Zaldúa, M. (2015). “Google News y el impacto de la Ley de Propiedad Intelectual en la prensa: un nuevo amanecer para la información”. Documentación de las Ciencias de la Información, Vol. 38: páginas. 67-81
\end{abstract}




\section{INTRODUCCIÓN}

Hace ya más de una década que los periodistas buscan un modelo informativo que les convierta en protagonistas de la noticia, como lo eran antes, que les permita seguir contando con el favor de los lectores. Hasta ahora no lo han logrado y tampoco se percibe en el horizonte una solución próxima. La pérdida de lectores en los periódicos -impresos y digitales-, diríamos de manera escandalosa, es proporcional al número de ellos que obtienen la información de las redes sociales o en otros sitios de internet que no se llaman periódicos.

La burbuja informativa estalló en España a finales de 2014, concretamente el 4 de noviembre, cuando las Cortes Generales deciden modificar el texto refundido de la Ley de Propiedad Intelectual, aprobado por Real Decreto Legislativo 1/1996, de 12 de abril, y la Ley 1/2000, de 7 de enero, de Enjuiciamiento Civil. Se trataba de un cambio significativo para los medios de comunicación, especialmente la prensa. Si bien la Ley contempla dos directivas de la Unión Europea, la realidad demuestra que ese es un paso incompleto, tal y como se comprobará luego al analizar la situación europea.

Ya hace tiempo que los editores vienen demandando cambios en la legislación. Ahora más necesarios a tenor de sus resultados económicos. Desde hace dos décadas empezaron a funcionar los resúmenes de prensa, que recortaban la compra de periódicos pues facilitaban a través de fotocopias los contenidos; pasando luego por lo que vino a llamarse los press clipping o recopilaciones de artículos/noticias/informaciones que luego se facilitaban a las empresas; hasta los RSS y los más polémicos que han empujado para que esta ley terminara por aplicarse, los llamados agregadores de noticias/agregadores de contenido.

El artículo 32 del Real Decreto Legislativo 1/1996, Citas y reseñas e ilustración con fines educativos o de investigación científica, en su apartado 1, venía recogiendo el funcionamiento de cómo afectaba esta ley a las publicaciones periódicas "Las recopilaciones periódicas efectuadas en forma de reseñas o revista de prensa tendrán la consideración de citas. No obstante, cuando se realicen recopilaciones de artículos periodísticos que consistan básicamente en su mera reproducción y dicha actividad se realice con fines comerciales, el autor que no se haya opuesto expresamente tendrá derecho a percibir una remuneración equitativa. En caso de oposición expresa del autor, dicha actividad no se entenderá amparada por este límite” (BOE, 04/11/2014).

La nueva ley modifica el título del artículo 32, así como su apartado 2, y se adicionan nuevos apartados 3, 4 y 5 . Aquí es donde los editores consideran clave el cambio y el inicio de la nueva polémica sobre los autores que producen la información, la manera en que se difunde y los dueños de contenidos. El texto señala: "La puesta a disposición del público por parte de prestadores de servicios electrónicos de agregación de contenidos de fragmentos no significativos de contenidos, divulgados en publicaciones periódicas o en sitios Web de actualización periódica y que tengan una finalidad informativa, de creación de opinión pública o de entretenimiento, no requerirá autorización, sin perjuicio del derecho del editor o, en su caso, de otros 
titulares de derechos a percibir una compensación equitativa. Este derecho será irrenunciable y se hará efectivo a través de las entidades de gestión de los derechos de propiedad intelectual. En cualquier caso, la puesta a disposición del público por terceros de cualquier imagen, obra fotográfica o mera fotografía divulgada en publicaciones” (BOE, 04/11/2014).

Otro artículo importante que afecta a los titulares de los derechos es el 138: Acciones y medidas cautelares urgentes. Se modifica y queda redactado en los siguientes términos: "El titular de los derechos reconocidos en esta ley, sin perjuicio de otras acciones que le correspondan, podrá instar el cese de la actividad ilícita del infractor y exigir la indemnización de los daños materiales y morales causados, en los términos previstos en los artículos 139 y 140. También podrá instar la publicación o difusión, total o parcial, de la resolución judicial o arbitral en medios de comunicación a costa del infractor" (BOE, 04/11/2014).

A pesar de estos cambios, esta Ley parece no haber contentado a casi nadie, excepto a los editores de grandes periódicos a través de la AEDE (Asociación de Editores de Diarios Españoles) que parecen ser los principales beneficiarios. Esta Asociación ya solicitó a Google en 2010 que pagara por el uso de la información. Un año después tomo parte activa en otras peticiones que se hicieron a nivel europeo. Los dos años siguientes fueron trabajando en la ley que se acaba de publicar para ir frenando el poder que acumulaba Google. Ya antes de que se promulgara la Ley existían diferentes posturas y sobre todo un futuro incierto porque la gran pregunta es ¿Por qué ya no hay lectores de noticias? o ¿Por qué los lectores prefieren informarse en otros lugares, (léase redes sociales)? La cuestión que nos planteamos es: ¿Estamos ante un nuevo amanecer para la información?

El objetivo de este artículo es analizar la situación a la que se enfrenta la prensa en España, comparándola con otros países europeos, tras la marcha de Google, que decidió cerrar Google News cuando se publicó dicha Ley. Además de ello, conocer cómo ha afectado de manera inmediata a los periódicos españoles. Todo ello teniendo en cuenta el uso que los lectores de medios hacen de las redes sociales en detrimento del periódico como fuente de información. Se pretende responder por tanto a las siguientes preguntas:-i ${ }^{2}$ Necesitan los lectores los periódicos impresos para estar informados, acuden a los periódicos digitales o se informan en las redes sociales? 


\section{LOS MEDIOS EN EL ENTORNO ACTUAL: CONSTRUYENDO EL FUTURO DEL PERIODISMO}

Nunca como en la última década se han publicado tantos libros y artículos de carácter premonitorio sobre el futuro del periodismo, sobre su muerte y usando el símil religioso su resurrección. En ese tiempo, el periodismo impreso se vio sobrepasado por el digital y éste por las redes sociales. $\mathrm{Y}$ en medio de todo ese proceso, los grandes "ubicuadores", acaparadores de lectores y depredadores de contenido se posicionaron al frente de la sociedad.

Es posible que todo esto sea una "cortina de humo" para tapar la verdadera realidad del periodismo: ya no hay lectores; o mejor dicho, los lectores emigraron a otros lugares para conseguir la información de actualidad.

El entorno actual de los medios se ha venido configurando desde comienzo del siglo, pero tiene un recorrido muy dinámico en: a) varias fechas (apariciones de redes sociales, fundamentalmente) que se han producido entre: 2004-2014; y b) autores que han señalado en sus obras los cambios más importantes del periodismo. El punto de partida sería 2004 con la aparición de Facebook que rompe un sistema de manejo de la información y el entretenimiento. Un año después se lanza YouTube y en dos más Twitter y Tumblr. En una década: 2004-14 (ver figura 1) se crearon las principales redes sociales que ahora están ofreciendo la información que antes buscaban los lectores en los periódicos.

Figura 1. Evolución de los Social Media 2004-2014

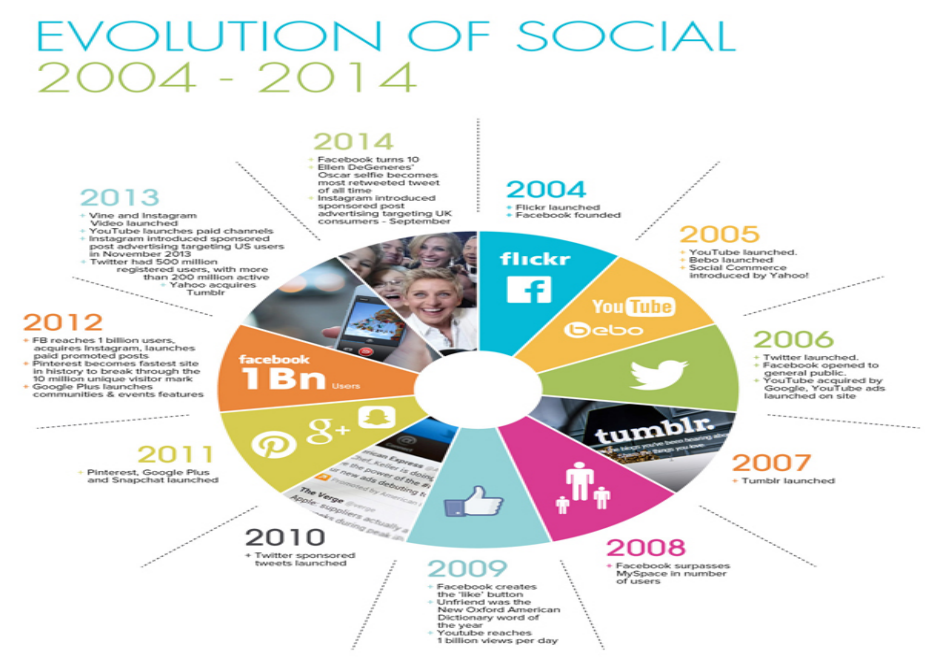

Fuente: MEDIAVIS10N 
Unos datos de finales de 2014, de la misma fuente que el gráfico (Kolovich, 2014), pueden avalar este punto de vista, sobre el uso que hasta 2014 se hicieron de las redes sociales: a) Tumblr: su servidor soportó más de 209.300.000 blogs y más de 94.9 mil millones de correos en total; b) Instagram: tiene 200 millones de usuarios activos y publican 60 millones de fotos al día, con una media de 1.6 mil millones diarios de "me gusta"; c) Twitter alcanzó ya los 284 millones de usuarios activos al mes y 500 millones de tweets se envían al día; d) Facebook ya tiene activos 1.32 mil millones de usuarios; e) LinkedIn: cuenta ya con 332 millones de usuarios registrados; f) Pinterest: tiene 70 millones de usuarios, de los cuales 40 son activos; g) YouTube: es la red que más está avanzando, pues cuenta en su haber más de mil millones de usuarios, 6 mil millones de horas de vídeo visto por meses y 100 horas de vídeo subidas por minuto; por último, h) Google: está presente en las redes sociales a través de más de mil millones de cuentas habilitadas y 359 millones de usuarios activos mensuales. Estos datos contrastan el crecimiento de éstas y otras redes frente al declive de los lectores en los periódicos, desde luego los impresos y ahora los digitales.

¿Qué medio/s puede/n sumar tantos usuarios? ¿Sería preciso que los periódicos se asociaran con estos sitios de internet? ¿Exclusión o inclusión? De cómo responsan los editores de prensa a estas preguntas dependerá el futuro de los medios. Desde luego, la nueva Ley de Propiedad Intelectual lo que viene a demostrar es que hay una fractura importante entre ambos contendientes y Google News ya ha tomado una decisión con resultados poco favorables a los medios españoles (Ver figuras 4 y 5 )

En segundo lugar, autores que vienen anunciando en los dos/tres últimos años ese movimiento. Sobre el "futuro del periodismo" hay cambios drásticos que están modificando virtualmente todos los aspectos que conocemos acerca de la emisión y recepción de información (Franklin 2012). En la misma línea, se analizan los resultados de la llamada revolución digital (Cerezo Gilarranz et. Al, 2012). Otros autores posicionaron a la prensa en su fase terminal (Díaz Nosty et. Al, 2013), con una visión global del periodismo desde la década de los setenta, pasando por el llamado periodismo electrónico hasta llegar al digital, o bien describen que Internet lejos de ser un problema puede y debe convertirse en el mejor aliado de los periodistas (Franco; Pellicer, 2014), o estudian la manera en que los medios deben plantear la información y la documentación cuando los lectores están emigrando a otros lugares a conseguirla (Marcos Recio, 2014; Marcos Recio, Fernández Sande, 2014).

En resumen, en los tres últimos años, la coincidencia de muchos autores sobre el futuro que espera a los periódicos va en la misma dirección: deben mejorar la información, dar protagonismo al usuario/lector y cambiar la dinámica de trabajo y la forma en que distribuyen los contenidos. 


\section{CREADORES DE CONTENIDO FRENTE A NUEVOS USUARIOS}

El cambio más importante hacia un nuevo amanecer informativo está en la creación de contenidos, sean o no puramente informativos. Hace tiempo que los lectores, la mayoría de ellos, se conformaban con leer el título y las entradillas de la información. Muy pocos profundizaban en el contenido. Pues bien, ahora les resulta más fácil porque esa manera de crear contenidos - un titular y varias líneas- es todo lo que desean los lectores y eso sí se lo ofrecen las redes sociales.

Lo que los editores deben entender en estos tiempos es la manera en que los lectores acceden a la información. Se enfrentan al menos a estos cuatro retos: a) Existe ya mucha información gratuita. Algunos usuarios colocan en Internet contenidos que se pueden considerar periodísticos. Otros lectores los reproducen y la cadena se amplía; b) El llamado periodismo ciudadano, aquél que se genera gracias a voluntarios que escriben y/o publican fotos de informaciones en las que se han visto implicados o conocen; por ejemplo, un ciudadano está cerca de un accidente de tráfico/ferroviario y ofrece las primeras fotografías e informaciones que a su vez se multiplican por las redes sociales. Los periodistas, la mayor parte de ellos, siguen creyendo que eso no es información, pero los lectores ya no irán a los periódicos; c) recomponer las plantillas y adecuarlas a los tiempos actuales. Los modelos de periódicos que funcionarán no son aquellos que cuenten con grandes plantillas, sino con grupos de redactores capaces de cubrir informaciones nacionales, pero al mismo tiempo dar la versión más próxima a lo que demandan los lectores; y d) contenidos cada vez más especializados. Se publicarán contenidos que los grandes periódicos dejan al margen y el éxito de esos medios radicará en que al ser más dinámicos y al hacer un periodismo más de calle, estarán ofreciendo los datos más próximos a los lectores.

El contenido que desean los nuevos lectores pasa por reubicar la información y lograr que interese de verdad al usuario/lector. Ya no es tan importante ofrecer una exclusiva, puesto que a los pocos segundos esa información ya está en los cinco continentes. Se trata, sobre todo, de mostrar contenidos que no cubren los grandes periódicos. Lo que se puede constatar ahora es que el modelo de periódico digital ya no es el referente informativo para los usuarios. La información más próxima a ellos la buscan en redes sociales. Mientras las grandes "rotativas digitales" no ofrezcan contenidos más próximos a los lectores, la información que actualizan cada pocos minutos no servirá para nada. 


\section{MIENTRAS ESPAÑA Y EUROPA CIERRA LAS PUERTAS... ESTADOS UNIDOS LAS DEJA ABIERTAS}

La mayoría de los editores siguen confiando en los periodistas como creadores de contenidos. Por su parte, las redes sociales encargan esa labor a determinados algoritmos que rastrean la red Internet en busca de información, entretenimiento, ocio, temas sociales, deportes, etc., que colocan luego en la zona visible y lo hacen en escasos segundos.

La pregunta clave sería: ¿Qué sucedería si nadie colocara contenidos en Internet? Pues que esos algoritmos no encontrarían nada. Pero eso ya no sucede. Este es el caballo de batalla entre los editores y los que defienden que otros sistemas rastreen la información y la ofrezcan a través de las redes sociales. La postura de los editores es clara: si creamos contenidos y ya nadie los lee en nuestro periódico no podemos atraer publicidad y, en consecuencia, el negocio se quiebra, por eso demandan a las empresas que usan su información para que paguen por esos servicios. Por su parte, las redes sociales que usan la información, especialmente Google News indica que ellos no ganan nada con esa información y además redirigen lectores desde su sitio. Eso supondría un gran beneficio para los editores.

\subsection{España y la nueva Ley de Propiedad Intelectual}

Google News decide cerrar en España. Mientras en algunos países europeos han mantenido una dura batalla, en otros ha negociado y en algunos ha pagado una parte de apoyo a la transformación digital de los periódicos. Antes de la Ley de Propiedad Intelectual ya existían movimientos entre ambas partes. Google News, a través de Eric E. Schmit, el CEO de la compañía anunció en un artículo publicado en El País, que "preferían la colaboración al conflicto", señalando que "cada mes Google hace que editores de todo el mundo reciban más de 10.000 millones de visitas" y eso se puede traducir en buena publicidad para los medios. Según el CEO de Google: "Cada clic es una oportunidad de negocio para aumentar la audiencia y generar ingresos. En 2013, solo a través de nuestro producto AdSense, proporcionamos 9.000 millones de dólares en ingresos a nuestros editores asociados" (Schmit, 2014).

Pero la colaboración no se produjo meses después. En España, la nueva Ley de Propiedad Intelectual establece el cobro de un canon (o impuesto) a los agregadores de noticias, si agregan sitios que formen parte de la Asociación de Editores de Diarios Españoles (AEDE). La compañía se defiende diciendo que Google News no tiene publicidad y que además revierte visitas a los medios. Además, indican que los periódicos pueden impedir que se haga ese rastreo, pero a cambio no saldrán sus enlaces en Google y perderán visibilidad (Herrera; Arboledas; Legeren 2014). Por su parte, la Asociación Española de Editores de Prensa Periódica (AEEPP) indicó en un comunicado que están dispuestos a adaptarse a los nuevos tiempos. 
No todos están de acuerdo con la postura que defiende la Asociación de Editores de Diarios Españoles (AEDE), incluso dentro de la propia profesión periodística se denuncia que es una maniobra de algunas empresas de periódicos que quieren tapar su mala gestión. Ya se ha creado una plataforma contra el canon de la AEDE: Coalición Prointernet porque daña las libertades y el desarrollo de la economía en Internet.

Así las cosas, a) los editores (algunos) creen que el gigante Google se hizo con su mercado publicitario y con la información; otros, esperan sacar un rendimiento de las negociaciones y hasta otros, los más pequeños, esperan acontecimientos que ayuden a mejorar la mala situación que atraviesan; b) periodistas, creadores de contenido: reciben un único pago por la información, a no ser que sean independientes. Aspiran a que su información les reporte más por lo que hacen y c) lectores: son cada vez más activos y quieren participar en los contenidos. Y todo ello en medio de una gran crisis periodística, ya que como se señala en el "Informe Anual de la Profesión Periodística 2014" los medios sufrieron recortes importantes, especialmente la TV: "En conjunto, en lo que va de año se destruyeron 2.412 puestos de trabajo (Figura 2), lo que representa el $20 \%$ del total desaparecido desde el comienzo de la crisis, hace siete años. Un porcentaje muy elevado, aunque algo menor al 29\% registrado un año antes (Palacio Llanos, 2014).

Figura 2. Destrucción de empleo en los medios de comunicación

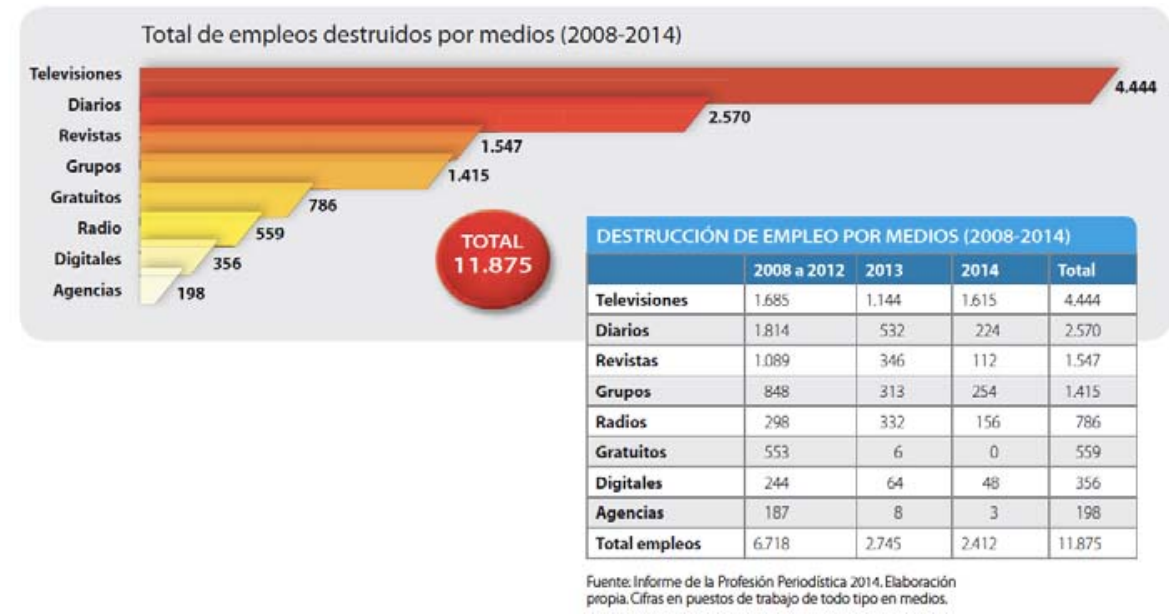

Fuente: Informe de la Profesión Periodística 2014 


\subsection{Países de Europa a favor y en contra del pago del canon}

Mientras que en España Google News echaba el cierre el 16 de diciembre, convirtiéndose así en el primer país en el que el buscador dejaba de prestar ese servicio, en otros países europeos el pulso entre editores de prensa y Google se ha mantenido con desiguales resultados: a) Inglaterra: a través de News Corp., de Rupert Murdoch, fue uno de los primeros en frenar este tipo de propuestas que ofrecía Google News y decidió crear algo similar en 2010: un agregador de noticias de pago que incluirá a los periódicos del grupo como The Times, The Sunday Times, además de ofrecer contenidos de otros editores; b) Bélgica: lo mismo que Inglaterra ha estado luchando en los tribunales contra el gigante Google. Después de varios años, se llegó a un acuerdo por el que incrementa su colaboración tecnológica y comercial con el sector, aunque sin contraprestación monetaria; c) Francia, también han mantenido una ardua disputa con Google respecto de los derechos de autor. El tema se resolvió, de forma parcial, cuando el CEO de Google, Eric E. Schmidt, firmó un acuerdo con el presidente francés, François Hollande, según el cual Google pagará a los editores de la prensa generalista francesa 60 millones de euros al año, y d) Alemania: con defensores y detractores que han ido cambiando de opinión en función de distintas situaciones del mercado financiero. Una de las más grandes editoriales de Europa, Axel Springer, cedió a la presión de Google después de que el tráfico a sus sitios web de Google se redujo en $40 \%$ y de Google Noticias en un $80 \%$. Por el contrario, hay editoriales que decidieron seguir con Google, como Frankfurter Allgemeine Zeitung y el Süddeutsche Zeitung.

El nuevo Comisario en la Unión Europea para asuntos digitales, Günther Oettinger, señaló que está decidido a introducir una ley de derechos de autor europea y una tasa sobre la propiedad intelectual y que esas nuevas leyes afectarían también a empresas con sede en territorio no europeo, con clara referencia a Google y otros buscadores.

\subsection{La otra cara de la moneda en Estados Unidos}

Los periódicos en Estados Unidos se dejan querer por lo que Google News y otros agregadores les facilitan, pero ellos están por la labor de ofrecer mejores informaciones y seguir contando con el respaldo de los lectores. Ni siquiera discuten estos temas. Eso le sucedió al Instituto Pointer, cuando recientemente hizo un estudio para saber la importancia que tenía Google News en algunos medios significativos como USA Today, Chicago Tribune o Los Ángeles Times, etc. Sin embargo, una parte importante de la tarta termina beneficiando a los periódicos que reciben importantes lectores a través de los enlaces que reenvían sitios como Google News (Figura 3). En el estudio se encontraron respuestas que señalan otras preocupaciones que tienen los periódicos en Estados Unidos: "La única estrategia es estar en la cima de las últimas noticias" dijo el portavoz de USA Today, Steve Kidera. Un responsable de The New York Times comentó que no estaba dispuesto a discutir si tenía una estrategia con 
Google News. Se trata de "tráfico orgánico", dijo el portavoz a Reuters, Heather Carpenter" (Beaujon, 2014). En definitiva, en Estados Unidos, no se discute ni se plantea esa lucha contra Google News; al contrario, se aceptan los usuarios/lectores que reciben a cambio de estar enlazados.

"En EE.UU se recibieron más de cien millones de visitas de Google News, entre enero y noviembre de este año (2014), casi un tercio de todo el tráfico de búsqueda de noticias al sitio" (Beaujon, 2014), lo que constata que es mejor ser aliado de Google News que estar contra él.

Figura 3. Principales vistas a los medios y porcentaje total de las mismas

\begin{tabular}{|c|c|c|c|}
\hline & SITE & $\begin{array}{l}\text { NEWS SEARCH VISITS } \\
\text { (JAN-NOV 2014) }\end{array}$ & $\begin{array}{l}\text { PERCENTAGE OF } \\
\text { TOTAL SEARCH VISITS }\end{array}$ \\
\hline 1 & usatoday.com & $104,104,800$ & $30.44 \%$ \\
\hline 2 & reuters.com & $84,681,000$ & $43.65 \%$ \\
\hline 3 & nytimes.com & $84,047,400$ & $19.08 \%$ \\
\hline 4 & dailymail.co.uk & $72,220,200$ & $7.88 \%$ \\
\hline 5 & nydailynews.com & $69,669,950$ & $29.71 \%$ \\
\hline 6 & foxnews.com & $69,631,200$ & $27.36 \%$ \\
\hline 7 & telegraph.co.uk & $65,805,850$ & $16.39 \%$ \\
\hline 8 & latimes.com & $63,150,000$ & $30.00 \%$ \\
\hline 9 & abcnews.go.com & $62,533,200$ & $25.42 \%$ \\
\hline 10 & washingtonpost.com & $61,123,650$ & $25.31 \%$ \\
\hline
\end{tabular}

Fuente: Instituto Poynter

\subsection{Google News y el valor de las noticias}

El negocio de Google no son las noticias. La compañía dejó claro desde el principio que ellos no cobraban nada por ese servicio y que estaban dispuestos a no pagar tampoco. Además, son conscientes de que con su motor de búsqueda ayudan a reenviar usuarios a los medios y que cuando se produjo el cierre en España a las dos horas del cierre, la caída de usuarios en los medios fue de dos dígitos. Así lo indicó Josh Schwartz, jefe del Servicio Analytics Chartbeart: "El servicio da seguimiento a unos 50 sitios, que van desde los medios de comunicación pequeños a los más grandes 
editores de periódicos, y mirando los datos muestran "una diferencia bastante masiva" en el tráfico en comparación con un día similar antes de la eliminación. En promedio, la caída es de entre 10 y 15 por ciento (Ingram, 2014). Como se observa en las Figuras 4/5, los sitios en español sufrieron una importante desaceleración en relación con el tráfico de enlaces pasadas 15 horas desde el cierre. Esa misma situación se resolvió en Alemania solicitando nuevamente la vuelta a los enlaces de Google News, pero en España era demasiado tarde porque la compañía había decidido salir del país, mientras que en Alemania se mantuvo.

Figuras 4/5. Tráfico de enlaces tras el cierre en España de Google News.
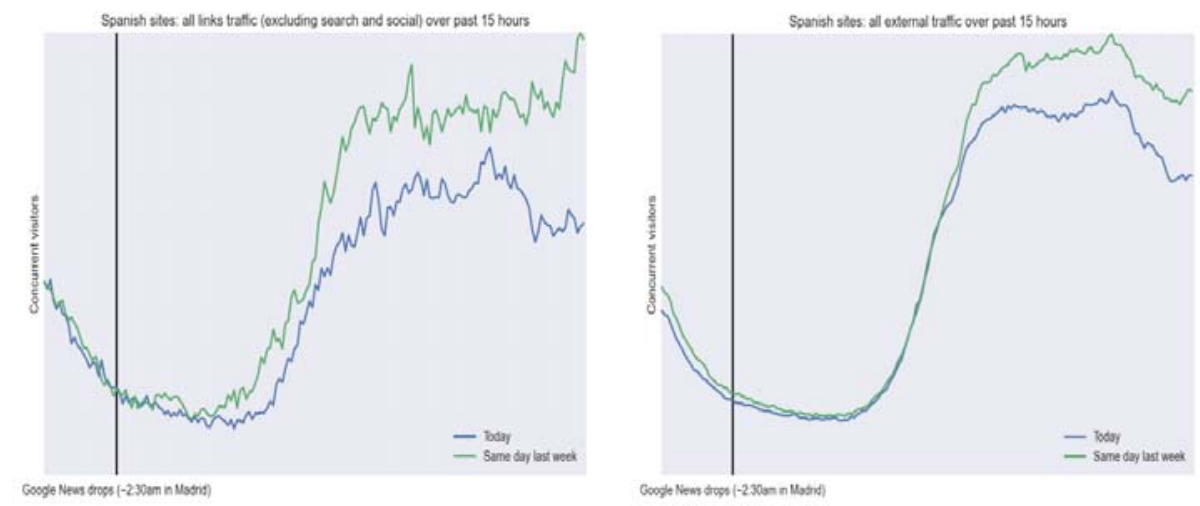

Fuente: Chartbeat

Google sigue siendo un importante controlador del tráfico desde que en 2002 puso en marcha Google News y los editores sabían que anotar un solo paso en Google News puede ayudar a conducir más tráfico que cualquier historia podría conseguir orgánicamente y, según Parse.ly, Google News envía un 35\% del tráfico de referencia de los editores (Bilton, 2014). Otras empresas señalan entre un 10-25\% del tráfico global de editores en Estados Unidos (Figura 6). 
Figura 6. Principales fuentes de referencia de tráfico

\section{TOP TRAFFIC REFERRAL SOURCES}

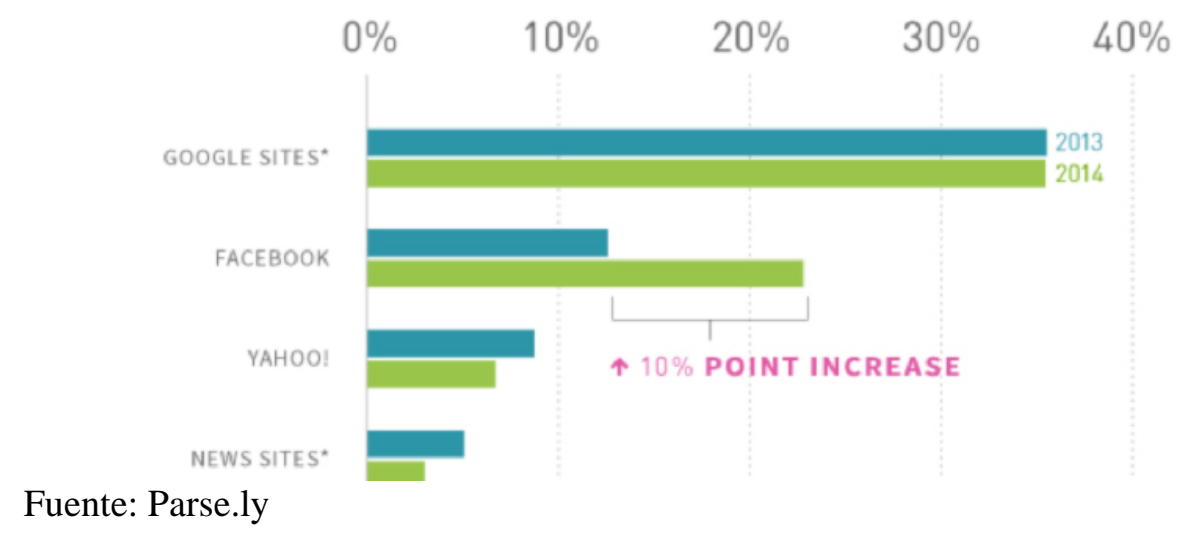

Algunos de los grandes sitios de Internet están pensando en el negocio de la información y eso sí será una competencia mortal con los editores que no acepten el trato. Ese será el nuevo amanecer de la información y el declive de los periódicos o su resurgimiento. Dos ejemplos avalan esta teoría:

a) A finales de noviembre de 2015 Google anunció que quiere ayudar a los lectores para que paguen por las noticias. Sería una manera directa de ayudar a los editores, al menos a los que sigan enlazados a Google News: "Google Contributor es una plataforma experimental que permite a las personas pagar a los editores para visitar un sitio. En lugar de comprar una suscripción, los lectores ponen entre 1 y 3 dólares al mes en una cuenta que se utiliza para pagar a los editores en función de cada visita. Actualmente, 10 sitios están participando en el experimento, incluyendo Mashable, The Onion, Science Daily, and wikiHow -otros no han sido anunciados por Google(Ellis, 2014).

b) Facebook quiere construir una información personalizada para cada uno de sus usuarios, con lo cual tendrían la información que necesitan sin tener que acudir a los periódicos. Una información publicada en Business Insider recogía las declaraciones de Mark Zuckerberg: "Nuestro objetivo es construir el periódico personalizado perfecto para cada persona en el mundo... Estamos tratando de personalizarlo y mostrarle las cosas que van a ser más interesantes para usted” (Eugene, 2014). 


\section{CONCLUSIONES}

El mundo avanza hacia un nuevo orden informativo. La producción y distribución de contenidos no es exclusiva de los periódicos. Ya nadie puede garantizar audiencias millonarias en los medios tradicionales. Solo las redes sociales reúnen a millones de personas interesadas en la información que reciben. No es una información completa, con declaraciones, con la visión de los expertos, pero la mayoría de los lectores la dan por buena.

No se conocen aún las razones que han llevado a Google a cerrar su sitio de noticias en España, cuando en otros países como Bélgica, Francia, Alemania, se enfrentaron a situaciones parecidas y resolvieron de otra manera. Queda clara la postura: No pagar. Y cuando lo hizo, como en Francia, no es un pago por el canon, sino una ayuda al trasvase de los medios hacia lo digital. ¿Es España un aviso para navegantes? Los editores han visto como el número de personas que acudían a través de Google News ha caído y no les interesa vivir sin ellos. Google también gana con más seguidores porque publicitariamente puede competir con otras redes sociales. En función de cómo se vaya aplicando la Ley de Propiedad Intelectual y los posibles acuerdos por parte de los editores, los periódicos y Google News seguirán trabajando juntos. No hay que olvidar que cualquiera puede evitar ser indexado por Google.

Tanto los editores españoles como Google News no han encontrado hasta la fecha un nexo común para negociar el uso de la información. La reforma de la Ley de Propiedad Intelectual ha sido la excusa para que ambas partes hayan renunciado a pactar sobre sus respectivos intereses. Google ofrece a los medios, ejemplos de cómo hacer publicidad, de cómo reinventarla y de cómo tener un compromiso y una lealtad mayor hacia sus usuarios. Aunque se han indicado propuestas que Google hace a los editores para que los usuarios paguen por la información, podría producirse una situación aún peor al cobrar a los editores por los vínculos que les envía (Jarvis, 2014).

En breve se producirán uniones de medios que se apoyarán en las redes sociales para acceder al gran público. Ya no se trata de hacer la mejor información, sino de facilitarla en tiempo y forma. La información de calidad, original, con autoridad, con la participación de los ciudadanos, considerando una mercancía de alto valor, se establecerá por periodistas y ciudadanos que consideran que el mundo sin información no tiene el mismo valor. Se necesitan cada vez más, noticias que aporten nuevos datos al mundo. 


\section{BIBLIOGRAFÍA}

BEAUJON, Andrew (2014). EE.UU. Hoy en día recibe una gran cantidad de tráfico de Google Noticias, 2014 (www.poynter.org/news/mediawire/307035/usa-todaygets-a-lot-of-traffic-from-google-news) Consulta: 16-04-2015.

BILTON, Ricardo (2014). Google Noticias: todavía es un controlador de tráfico importante, 2014 (http://digiday.com/publishers/google-news-still-major-trafficdriver/) Consulta: 15-04-2015.

BOE. Núm. 97, de 22 de abril de 1996. Real Decreto Legislativo 1/1996, de 12 de abril.

BOE. Ley 21/2014, de 4 de noviembre, por la que se modifica el texto refundido de la Ley de Propiedad Intelectual, aprobado por Real Decreto Legislativo 1/1996, de 12 de abril, y la Ley 1/2000, de 7 de enero, de Enjuiciamiento Civil.

CEREZO GILARRANZ, D. et al. «El futuro del periodismo», Cuadernos de comunicación Evoca, n ${ }^{\circ}$ 7, 2012, pp.3.

DÍAZ NOSTY, B. et al. La prensa en el nuevo ecosistema informativo. iQue paren las rotativas!. México: Ariel, 2013

ELLIS, Justin. ¿Por qué Google está ofreciendo otra oportunidad para ayudar a los lectores a pagar por las noticias? 2014 (www.niemanlab.org/2014/11/why-googleis-taking-another-shot-at-helping-readers-pay-for-news/) Consulta: 15-04-2015.

FRANCO, M; PELLICER, M. Optimismo para periodistas. Claves para entender los nuevos medios de comunicación en la era digital. Barcelona: UOC, 2014.

FRANKLIN, Bob. The future of journalism. Londres, Routledge, 2012.

HERRERA SOLANA, Víctor; Arboledas, Luis; Legeren Álvarez, Elisa. «Universidades y Google News: visibilidad internacional a través de los medios de comunicación online”, Revista Española de Documentación Científica, v. 37, n 3, 2014 .

http://dx.doi.org/10.3989/redc.2014.3.1130

INGRAM, Mathew. El tráfico externo a los sitios de noticias en español se desploma después de que Google se cerró, 2014 ( https://gigaom.com/2014/12/16/traffic-tospanish-news-publishers-plummets-after-google-move) Consulta: 16-04-2015.

JARVIS,Jeff. ¿QQué podría hacer Google por las noticias?, 2014 (https://medium.com/whither-news/what-could-google-do-for-news-a6eab208c4b1) Consulta: 15-04-2015.

KOLOVICH, Lindsay. La evolución de los medios sociales desde el año 2004. Infografía, 2014 (www.mediavisioninteractive.com/blog/social-media/evolutionsocial-media) Consulta: 15-04-2015.

KIM, Eugene. Mark Zuckerberg Wants To Build The 'Perfect Personalized Newspaper' For Every Person In The World, 2014 (www.businessinsider.com/markzuckerberg-wants-to-build-a-perfect-personalized-newspaper-2014-11) Consulta: 13-04-2015. 
MARCOS RECIO, Juan Carlos. «Otra forma de plantear la información y documentación en los medios digitales», Revista General de Información y Documentación, v. 24, n. 1, 2014, pp. 119-134

http://dx.doi.org/10.5209/rev_RGID.v24n1.45467

MARCOS RECIO, Juan Carlos; Fernández Sande, Manuel. «Abriendo camino en los entornos digitales de comunicación: algunas propuestas significativas», Documentación de las Ciencias de la Información, v.37, 2014 pp. 231-254

http://dx.doi.org/10.5209/rev_DCIN.2014.37.46825

PALACIO LLANOS, Luis. Informe Anual de la Profesión Periodística 2014. Asociación de la Prensa de Madrid (http://www.apmadrid.es/publicaciones/informeanual-de-la-profesion-periodistica) Consulta: 15-04-2015.

SCHMIT, Eric E. «Preferimos la colaboración al conflicto». El País, 10 abril, 2014 (http://elpais.com/elpais/2014/04/09/opinion/1397053507_198997.html) Consulta: 15-04-2015. 